\title{
Das Eherecht in der Republik Österreich 1918-1978*
}

This article deals with the modifications of the "Austrian monarchical" matrimonial law (divided into three religious denominations; no compulsory civil marriage; patriarchal, committed to a middle-class family model; moulded on denominational ideals; obvious in the absence of a law of divorce for Roman Catholics) from 1919 to 1978. Discussions about reform proved without results until 1938 and the so-called "Sever-Ehen" led to the frequently quoted "Marriage Chaos" during the First Republic. The introduction of the German matrimonial law resulted in an important move towards modernisation (non-denominational matrimonial law; compulsory civil marriage). Further discussions about change proved successful only after the abolition of the conservative-Catholic and authoritariansocialistic paradigm (fundamental reform of Family Law).

\section{Einleitung}

Grundsätzlich steht das Eherecht in den meisten europäischen Staaten im Mittelpunkt eines Säkularisierungs- und Modernisierungsparadigmas, dessen Referenzrahmen die Entflechtung von Kirche und Staat als wichtiger Aspekt einer funktionalen Differenzierung ist. Die österreichischen Ehediskurse weisen aber eine Besonderheit auf, denn es kam zu einer Verlängerung dieses Prozesses bis in die 60er Jahre des 20. Jahrhunderts. Die These vom 19. Jh. als einem zweiten konfessionellen Zeitalter (O. Blaschke), das - auf Österreich umgelegt - mit den 60er Jahren des 20. Jh. endet, entfaltet einen beträchtlichen heuristischen Nutzen. ${ }^{1}$

\footnotetext{
* Dieser Aufsatz ist Ursula Floßmann zur Emeritierung gewidmet.

${ }^{1}$ Vgl. BlaschKe, Das 19. Jahrhundert, 38-75; DERS., Konfessionen im Konflikt; als Einstieg in die vielschichtige "Säkularisierungsdebatte" vgl. die Beiträge zum Abschnitt „Säkularisierung, Subtraktion und Transzendenz", in: KÜHNLEIN, BACHMANN, Neue Perspektiven 547; vgl. weiters die Beiträge zum Rahmenthema „Säkularisierung und Neuformierung des
}

\section{Eherechtsentwicklung 1918-1933}

$\S 16$ Staatsgründungsbeschluss vom 30. Oktober 1918 lautet: „Insoweit Gesetze und Einrichtungen, die in den im Reichsrate vertretenen Königreichen und Ländern in Kraft stehen, durch diesen Beschluss nicht aufgehoben oder abgeändert sind, bleiben sie bis auf weiteres in vorläufiger Geltung."2

Während sich die Republik Deutschösterreich auf der Ebene des Verfassungsrechts eine neue Ordnung gab, übernahm die Provisorische Nationalversammlung durch $\S 16$ unterhalb der Verfassungsebene grundsätzlich die Rechtsord-

\footnotetext{
Religiösen. Gesellschaft und Religion in der zweiten Hälfte des 20. Jahrhunderts“, in: Archiv für Sozialgeschichte 51 (2011), insbesondere den Einleitungsbeitrag von ZIEMANN, Säkularisierung und Neuformierung 3-36.

2 Beschluss der Provisorischen Nationalversammlung für Deutschösterreich über die grundlegenden Einrichtungen der Staatsgewalt, StGBl. 1918/1.
} 
nung Cisleithaniens - materielle Kontinuität bei formeller Diskontinuität.

\section{1. Das „altösterreichische" Eherecht}

Was hatte sich die Republik mit der "materiellen Kontinuität" im Bereich des Eherechts eingehandelt?

Übernommen wurde das sogenannte „altösterreichische Eherecht", eingehandelt hatte man sich eine - rechtsvergleichend betrachtet - familienrechtliche Modernitätsverzögerung inklusive engagierter, aber ergebnislos gebliebener Reformdiskurse.

Das altösterreichische Eherecht ist ein Produkt des neuzeitlichen Ausdifferenzierungsprozesses von Staat und Kirche. Der grundlegende Wandel wurde durch Joseph II. vollzogen, der auf der Folie der Distinktionstheorie - Trennung zwischen Ehesakrament und Vertrag - im Ehepatent 1783 die staatliche Hoheit für den Vertrag beanspruchte. ${ }^{3}$ Die Grundsätze des Josephinischen Eherechts wurden in das ABGB 1811 übernommen und mündeten in ein dreifach gegliedertes Eherecht für Katholiken, "nicht katholische christliche Religionsverwandte" und für Juden. ${ }^{4}$

Was sind die prägenden Leitlinien?

- Das Eherecht war ein patriarchalisches, dem bürgerlichen Familienmodell verpflichtet, ein Befund, der sich aus der Zusammenschau von Rechtswirkungen der Ehe wie des Ehegüterrechts unmissverständlich ergibt. ${ }^{5}$

- Keine obligatorische Zivilehe

„Die feyerliche Erklärung der Einwilligung” - so $\S 75$ ABGB - "muß vor dem ordentlichen Seel-

\footnotetext{
${ }^{3}$ Grundlegend: CONRAD, Grundlegung der modernen Zivilehe 336; DERS., Staatliche Theorie und kirchliche Dogmatik 1172.

${ }^{4}$ LEISCHING, Römisch-katholische Kirche in Cisleithanien 10; KALB, Kirchliches Ehenichtigkeitsverfahren 722; FloßMANN, Privatrechtsgeschichte 81.

${ }^{5}$ LEHNER, Familie - Recht - Politik 17.
}

sorger eines der Brautleute, er mag nun, nach Verschiedenheit der Religion, Pfarrer, Pastor oder wie sonst immer heißen oder von dessen Stellvertreter in Gegenwart zweyer Zeugen geschehen". Wenn auch die Seelsorger als Staatsorgane handelten, wurde dadurch keine von der Kirche unabhängige Form der Eheschließung geschaffen. ${ }^{6}$

Das Eherecht ist materiell einem kirchlich bestimmten Leitbild verpflichtet, dramatisch sichtbar im Scheidungsrecht. ${ }^{7}$

Das ABGB unterschied zwischen Scheidung von Tisch und Bett (§§ 103ff.) und der Ehetrennung (§§ 115ff.). Mit der Scheidung wurde nur die häusliche Gemeinschaft aufgelöst, eine Wiederverheiratung war nicht möglich ${ }^{8}$. Demgegenüber bewirkte die Trennung die Auflösung des Ehebandes und ermöglichte das Eingehen einer weiteren Ehe. ${ }^{9}$

\footnotetext{
${ }^{6}$ Vgl. LeHNHOFF, in: Klang 500; FuHRMANN, Einführung der fakultativen Zivilehe 188.

${ }^{7}$ Vgl. LEHNER, Familie - Recht - Politik, 38; SCHAUER, Recht der Ehescheidung 20-63; FUHRMANN, Einführung der fakultativen Zivilehe,183.

${ }^{8}$ Die Scheidung erfolgte durch gerichtliches Verfahren nach vorgängiger dreimaliger Versöhnungstermine vor dem Pfarrer. Das ABGB unterscheidet zwischen einvernehmlicher Scheidung (§§ 103ff.) und ohne Einverständnis (§§ 107ff.). Einvernehmliche: notwendig war die Erklärung der Ehegatten vor Gericht über ihre Einigung bezüglich Scheidung, Vermögen und Unterhalt; Ohne Einverständnis: an Gründe gebunden (demonstrative Aufzählung): Ehebruch, Verurteilung wegen eines Verbrechens, boshaftes Verlassen, unordentlicher Lebenswandel, gefährliche Nachstellung, schwere Misshandlungen sowie empfindliche wiederholte Kränkungen und anhaltende, mit Gefahr der Ansteckung verbundene „Leibesgebrechen“. Klageberechtigt war nur der gekränkte Ehegatte.

${ }^{9}$ Die Trennung einer Ehe zwischen „nichtkatholischen christlichen Religionsverwandten" war an das Vorliegen von Gründen gebunden (taxative Aufzählung): Ehebruch, Verurteilung wegen Verbrechens zu einer mindestens fünfjährigen Kerkerstrafe, boshaftes Verlassen, Lebensnachstellung, wiederholte schwere Misshandlung. Daneben war auch eine „einverständ-
} 
Eine Ehetrennung wurde aber nur gestattet, wenn die Ehegatten zum Zeitpunkt der Eheschließung nicht-katholische Christen oder Juden waren - damit war die absolute Unauflöslichkeit der Katholikenehe besiegelt, zusätzlich noch verstärkt durch das Impedimentum catholicismi: Wurde die Ehe von „nichtkatholischen christlichen Religionsverwandten" getrennt, so konnten diese zu Lebzeiten des anderen Ehegatten nur mehr Nichtkatholiken heiraten, überdies war die Wiederverheiratung bei Lebzeiten des ersten Gatten überhaupt für jenen Eheteil ausgeschlossen, der nach Eingehung der getrennten Ehe katholisch geworden war. ${ }^{10}$

Das „altösterreichische Eherecht" ist aber nicht vollständig charakterisiert, erinnert man nicht auch an die Verbindung von Thron und Altar im Neoabsolutismus, eine Strategie, die auch noch in der Republik fortwirkte.

Kaiser Franz Joseph, reserviert gegenüber demokratischen oder konstitutionellen Legitimationen, stützte sich im Neoabsolutismus auch auf die Katholische Kirche, ${ }^{11}$ sichtbarer Ausdruck ist das - schon damals anachronistische - Konkordat $1855 .{ }^{12}$

Art. X sieht die Übertragung der Jurisdiktion über die Ehesachen an den kirchlichen Richter vor, der hierüber „nach Vorschrift der Heiligen Kirchengesetze ... zu urtheilen und nur die bürgerlichen Wirkungen der Ehe an den weltlichen Richter zu verweisen (hat)“. In der Durchführungsgesetzgebung wurde das kanonische Eherecht für den staatlichen Bereich eingeführt und

liche Trennung“ wegen „unüberwindlicher Abneigung" vorgesehen. Hier konnte der Richter zunächst auf Scheidung von Tisch und Bett erkennen.

${ }^{10}$ Authentische Interpretation durch die Hofdekrete vom 26. 8. 1814 (JGS Nr. 1099) und vom 17.7.1835 (JGS Nr. 61); vgl. LENHOFF, in: KLANG, 451.

${ }^{11}$ RUMPLER, Eine Chance für Mitteleuropa 341.

12 Konkordat vom 18. 8. 1855, RGBl. 195; vgl. WEINZIERL-FISCHER, Österreichische Konkordate 60; PRIMETSHOFER, Ehe und Konkordat 9; LEISCHING, Römisch-katholische Kirche in Cisleithanien 25. die eherechtlichen Bestimmungen des ABGB für Katholiken außer Kraft gesetzt. Die kirchlichen Gerichte übten an Stelle des Staates die Ehejurisdiktion aus und entschieden auch für den staatlichen Bereich endgültig. ${ }^{13}$

Diese restaurative Regelung blieb aber Episode, denn in der kultuspolitischen Durchführungsgesetzgebung zur Dezemberverfassung 1867, den sog. Maigesetzen 1868 - eines betraf die Ehe wurden die Vorschriften des zweiten Hauptstückes des ABGB über die Ehe der Katholiken wieder in Kraft gesetzt und die Gerichtsbarkeit in Ehesachen der Katholiken den weltlichen Gerichtsbehörden überwiesen. Darüber hinaus wurde auch die sog. "Notzivilehe“ eingeführt. Sollte der katholische Seelsorger die Vornahme der Trauung auf Grund eines kanonischen vom Staat nicht anerkannten Hinderungsgrundes verweigern, wurde es den Nupturienten freigestellt, ihre Eheerklärung vor der politischen Behörde abzugeben (,absolute Notzivilehe $\left.{ }^{\prime \prime}\right) \cdot{ }^{14}$ Erweitert wurde diese Regelung durch ein Gesetz vom 9. April 187015, wodurch die zivile Eheschließung für Personen eingeführt wurde, die keiner gesetzlich anerkannten Kirche oder Religionsgesellschaft angehören („,relative Notzivilehe").

Weitergehende Reformen im Hinblick auf eine grundsätzliche Umstrukturierung des Eherechts scheiterten, alle Bemühungen um eine Ehereform $^{16}$ blieben ohne Ergebnis.

${ }^{13}$ Am 8. 10. 1856 erging das kaiserliche Patent über die Ehen der Katholiken in Österreich und die Anweisung für die geistlichen Gerichte des Kaisertums Österreich in Betreff der Ehesachen (RGBl. 185); vgl. РототSCHNIG, Staatlich-kirchliche Ehegesetzgebung im 19. Jahrhundert 401.

${ }^{14}$ Gesetz vom 25. 5. 1868, RGBl. 47.

${ }^{15}$ Gesetz vom 9. 4. 1870, RGBl. 51.

${ }_{16}$ Bemerkenswert die Enquete der Kulturpolitischen Gesellschaft, an der sich auch Sigmund Freud mit einer Stellungnahme beteiligte. Eine Forderung nach Gleichberechtigung beider Geschlechter lehnte er ab, denn die "Gleichberechtigung der Geschlechter schliesst sich geradezu aus durch ihre verschiedenar- 
Da auch die Teilnovellen zum ABGB das Eherecht ausklammerten, die bereits 1875 im Deutschen Reich eingeführte Zivilehe damit keine Berücksichtigung fand, wurde der überkommene status quo bis zum Untergang der Monarchie beibehalten. Der „Umgehungsweg“ mittels der sog „Siebenbürger“ bzw. den „Ungarischen Ehen" war auf Grund des damit verbundenen finanziellen Aufwandes nur begüterten Personen möglich, überdies anerkannte die österreichische Rechtsprechung auf Nichtanerkennung derartiger ,"aus Ungarn geschöpfter Urteile“. ${ }^{17}$

Bemerkenswerter Weise wurde im Reformdiskurs das Anliegen einer grundsätzlichen Gleichstellung von Mann und Frau im Eherecht durch die Zentralforderungen nach der Zivilehe und Trennbarkeit der Katholikenehe überlagert.

Dieser Befund gilt aber nicht für die alte Frauenbewegung. ${ }^{18}$ Die Leistungen der österreichischen Sozialdemokratinnen für eine gesellschaftliche Emanzipation wurden mehrfach gewürdigt, aber auch die bürgerliche Frauenbewegung kann nicht nur auf systemimmanente Weiterentwicklungswünsche reduziert werden. Neben der Einführung der Zivilehe und der Ehetrennung für katholische Ehegatten wurden auch etwa die gesetzliche Anerkennung des Konkubinates, der Unterhaltsanspruch für die Konkubine gefordert, weiters wurden Reformen zu-

tige Rolle in der Fortpflanzungsfunktion“, bezüglich der Unauflöslichkeit der Ehe hält er fest, dass diese „bedeutenden ethischen und hygienischen Grundsätzen und psychologischen Erfahrungen" widerspreche, vgl. BOYER, Freud, Marriage, and late Viennese Liberalism 72, Abdruck der Stellungnahme, 99.

${ }_{17}$ PeILIKAN, Aspekte der Geschichte des Eherechts in Österreich, 195; LEHNER, Familie - Recht - Politik 70. Keine Umgehung waren die sog "Salzburger Ehen“. Hier handelte es sich um eine Einsegnung von Verbindungen gerichtlich geschiedener Katholiken durch den katholischen Priester, antiklerikalen Romanschriftsteller und Aktivist der „Los-von-RomBewegung“ Johann Kirchsteiger, vgl. RINNERTHALER, Johann Kirchsteiger 823.

${ }^{18}$ FLOBMANN, Frauenrechtsgeschichte 131. gunsten der Versorgung der schuldig geschiedenen Frau und Änderungen im Ehegüterrecht, wie z.B. die Beseitigung der einschlägigen Rechtsvermutungen zugunsten eines Verwaltungs- und Nutzungsrechts des Ehemannes, eingemahnt. ${ }^{19}$

Ohne dies zu vertiefen: Die Reformbestrebungen waren erfolglos und resümierend ist festzuhalten: Die Republik übernahm ein patriarchalisches, einem bürgerlichen Familienmodell verpflichtetes Eherecht, das eines der zentralen Themen in der Kampfzone der Säkularisierung der politischen Kultur war.

\section{2. Das Eherechtschaos in der 1. Re- publik}

Auf dem Hintergrund der kultuspolitischen Bruchlinien gingen die Auseinandersetzungen in der 1. Republik ungebrochen weiter. Albert Sever brachte bereits am 27. November 1918 einen Ehereformantrag in die Provisorische Nationalversammlung ein, der u.a. die Trennbarkeit der Ehen unabhängig vom Religionsbekenntnis vorsah. ${ }^{20}$ Kurz darauf erneuerte der von sozialdemokratischer Seite unterstützte parteilose Abgeordnete Julius Ofner seinen bereits 1908 und nach Wiedereinberufung des Reichsrates 1917 eingebrachten Antrag auf Einführung der obligatorischen Zivilehe und Führung der Geburts-, Ehe- und Sterberegister durch die Gemeindebehörden. ${ }^{21}$ Beide Anträge wurden dem Justizausschuss zugewiesen, überdies ermächtigte der Staatsrat den deutschnationalen Staatssekretär für Justiz Julius Roller zur Ausarbeitung eines Entwurfes mit der Maßgabe, die Trennungsgründe des $\S 115$ ABGB mit Ausnahme des Trennungsgrundes der „unüber-

\footnotetext{
${ }^{19}$ Vgl. HeIndL, Frau und bürgerliches Recht 134; FRYSAK, Legale Kämpfe 65.

${ }^{20}$ Beil. 54 der Sten. Prot. der Prov. NV von 1918 und 1919.

${ }^{21}$ Beil. 75 der Sten. Prot. der Prov. NV von 1918 und 1919.
} 
windlichen Abneigung" auf katholische Ehen anzuwenden, aber von der Einführung der obligatorischen Zivilehe und einer Verstaatlichung der Registerführung abzusehen. Dieser Entwurf wurde vom Justizausschuss mit großer Mehrheit angenommen, fand aber keine Mehrheit in der Provisorischen Nationalversammlung. ${ }^{22}$ Unüberhörbar waren in der Debatte auch antisemitische Untertöne und die Reichspost stellte nach der Abstimmungsniederlage mit Genugtuung fest, die "Judenreform" sei abgelehnt worden, die "Ehezerstörung" verhindert. ${ }^{23}$

Den Anliegen des engagierten und auch öffentlich wirksamen österreichischen Eherechtsreformvereins verpflichtet versuchte auch die unermüdliche Adelheid Popp mit einem trickreichen Gesetzesentwurf Änderungen zu erwirken, scheitere aber an den Mehrheitsverhältnissen der Konstitutionierenden Nationalversammlung. Ihr Vorschlag ließ zwar die konfessionelle Grundlage des Eherechts unangetastet, stellte aber für die „Trennbarkeit einer Ehe dem Bande nach" auf das Religionsbekenntnis des Trennungswerbers zum Zeitpunkt der Einbringung des Trennungsbegehrens und nicht wie bisher auf das Bekenntnis zur Zeit der Eheschließung

\footnotetext{
${ }^{22}$ Vgl. Beil. 145 der Sten. Prot. der Prov. NV von 1918 und 1919; vgl. LEHNER, Familie - Recht - Politik, 104; PRIMETSHOFER, KREMSMAIR, Gesetzliche Entwicklung 449; vgl. KREMSMAIR, Eherechtsreformversuche 524537.

${ }^{23}$ HARMAT, Ehe auf Widerruf 88; vgl. auch die Charakterisierung der Niederlage der Ehereformer durch Ignaz Seipel, einen Tag nach der Abstimmung, in einem Brief an Heinrich Lammasch (25. 1. 1919): „Der Sieg der Ehereformfrage wird eine Anzahl Stimmen kosten, da es doch sehr viele brüchige Ehen gibt. Aber er ist ein Sieg des Prinzips und hat die Sozialdemokraten mit den Juden isoliert. Die Freude in unseren Frauenkreisen ist groß. Natürlich wird sich auch die definitive Nationalversammlung mit dieser Frage wieder beschäftigen müssen, die uns schwere Arbeit machen wird.", zitiert nach WEINZIERL-FISCHER, Konkordate 155 .
}

ab. ${ }^{24}$ Danach wären Katholikenehen zwar grundsätzlich auch weiterhin untrennbar, aber ein Austritt eröffnete die Trennungsmöglichkeit. Außerparlamentarische Bemühungen wie auch parlamentarische Vorstöße blieben jedoch erfolglos, denn im Oktober 1919 gingen die christlichsoziale Partei und die Sozialdemokraten eine Koalition ein und vereinbarten im Koalitionspakt das Verhältnis von Kirche und Staat einschließlich der Ehegesetzgebung im Rahmen der neuen Verfassung zu regeln. ${ }^{25}$

Der sozialdemokratische Entwurf forderte mit dem plakativen Postulat einer Trennung von Staat und Kirche die Beseitigung der Religion aus dem Eherecht (obligatorische Zivilehe, konfessionsneutrales Ehetrennungsrecht), ${ }^{26}$ demgegenüber forderten die Christlichsozialen die Anerkennung des kirchlichen Rechts für Katholikenehen durch den Staat ${ }^{27}$ - eine Einigung war nicht möglich.

Auch im Koalitionsvertrag vom Mai 1922 zwischen Christlichsozialen und Großdeutschen wurden die „kulturellen Angelegenheiten” dazu zählte auch die Eherechtsreform - zurückgestellt, sozialdemokratische Reformversuche blieben regelmäßig erfolglos. ${ }^{28}$

Die „Eherechtsreform” scheiterte an der jeweiligen Koalitionsräson und wurde auf den administrativen Weg verschoben, das Drama der Dispensehen eskalierte. ${ }^{29}$ Ausgangspunkt war

${ }^{24}$ Beil. 370 der Sten. Prot. der Konst. NV vom 30. 7. 1919; vgl. OfNER, Antrag Popp 257f.

${ }^{25}$ ERMACORA, Entstehung der Bundesverfassung 14 Anm. 77 (Abdruck der Koalitionsvereinbarung).

${ }^{26}$ ERMACORA, Entstehung der Bundesverfassung 571 (Art. 128, 129, 135).

${ }^{27}$ Vgl. Parteiprogramm der Wiener Christlichsozialen Partei 1919, II (Kulturforderungen) 2, abgedruckt bei BERCHTOLD, Österreichische Parteiprogramme 365 .

${ }^{28} \mathrm{Vgl}$. HARMAT, Ehe auf Widerruf $111 \mathrm{f}$.

${ }^{29} \mathrm{Vgl}$. HARMAT, Ehe auf Widerruf (grundlegend; vgl. SCHWARZ, Rezension); KALB, Dispensehen 273-292; NESCHWARA, Kelsen als Verfassungsrichter 353; DERS., Hans Kelsen und das Problem der Dispensehen, 246. 
das in $\S 83$ ABGB verankerte landesfürstliche Gnadenrecht zur Dispensation von Ehehindernissen: „Aus wichtigen Gründen kann die Nachsicht von Ehehindernissen bei der Landesstelle angesucht werden, welche nach Beschaffenheit der Umstände sich in das weitere Vernehmen zu setzen hat."

Bereits in der Monarchie wurde in - allerdings nur wenigen - Einzelfällen und primär zugunsten von Antragstellern begüterter Schichten auf der Grundlage des § 83 ABGB vom Ehehindernis des bestehenden Ehebandes dispensiert und damit die Möglichkeit einer zweiten Ehe, einer sog „Dispensehe” geschaffen.

Nachdem die Sozialdemokraten 1919 in Niederösterreich die Mehrheit erlangten, benutzte der niederösterreichische Landeshauptmann Albert Sever die Dispensationsbefugnis des $\S 83$ ABGB systematisch und erteilte zahlreichen geschiedenen Katholiken mit der Nachsicht vom Ehehindernis des Ehebandes die Möglichkeit zur Wiederverehelichung (,Severehen").

Christlichsoziale Landesregierungen weigerten sich, derartige Dispensen zu erteilen, doch erlangten die von ihnen abgewiesenen Dispenswerber im Rekursweg die gewünschte Dispensehe vom Staatsamt (Ministerium) des Inneren (bzw Bundeskanzleramt).

Die Zahlen dieser „Eherechtsreform im Verwaltungswege" sprechen eine beredte Sprache: 1921 rühmte sich Sever, in Niederösterreich 15.000 Dispensehen gestiftet zu haben, bis zum Jahre 1929 sind 55.000 Dispensen vom Hindernis des Ehebandes erteilt worden, bis 1935 wird von ca. 70.000 ausgegangen. ${ }^{30}$

Die juristische Bewertung dieser Praxis war allerdings umstritten und äußerst kontrovers. Der zentrale Punkt betraf die Gültigkeit der nachfolgenden „Dispensehe”. Ist das impedimentum ligaminis einer Dispens zugänglich? Wird das Eheband der ersten Ehe durch die Dispens,

\footnotetext{
${ }^{30}$ HARMAT, Ehe auf Widerruf 487, 248 Anm. 33.
}

durch den nachfolgenden Eheabschluss oder überhaupt nicht aufgelöst? Führt die Dispensehe $\mathrm{zu}$ einem gleichzeitigen Bestehen zweier Ehen ein und desselben Partners? Welcher Gattin gebühren dann Unterhalts-, Pensions- und Erbansprüche?

Am 19. März 1921 hob der VwGH eine vom Staatsamt des Inneren und Unterricht erteilte Dispens als "gesetzlich nicht begründet" auf und verwies darauf, dass die Auflösung einer Ehe in die Kompetenz der Gerichte falle. ${ }^{31}$

Diese Auffassung teilte auch der OGH. 1921 nahm er in einem Gutachten zur Gültigkeit Stellung. Danach hatte weder die Dispens noch die allfällige zweite Ehe Einfluss auf den Bestand der Erstehe. Die zweite Ehe wurde jedoch nicht als "Nichtehe" qualifiziert, sondern als ungültige Ehe, die solange Bestand hatte, bis deren Ungültigkeit gerichtlich festgestellt worden war. ${ }^{32}$ Die nun zahlreich erfolgten Ungültigkeitserklärungen der Zweitehe durch die Zivilgerichte führten zu einem „Ehewirrwarr”, zu einer Eherechtsunordnung. „Derselbe Staat, der durch seine Verwaltungsbehörde die Schließung der Ehe ausdrücklich erlaubte, erklärte durch seine Gerichte eben diese Ehen für ungültig. Die Autorität des Staates konnte kaum in ärgerer Weise erschüttert werden" (H. Kelsen) - bzw. plakativer formuliert: „Im Hexameter segnet der Landesbeamte das Brautpaar; Im Pentameter darauf, reißt das Gericht es entzwei". ${ }^{33}$

1927 bejahte der VfGH auf Vorschlag seines ständigen Referenten Kelsen einen positiven Kompetenzkonflikt und sah bei der Beurteilung eines Verwaltungsaktes - auch nur als Vorfrage, um die Gültigkeit einer Ehe zu entscheiden einen unzulässigen Eingriff in die Kompetenz der Verwaltungsbehörden. Mit dieser Bewertung eines Bindungskonfliktes als indirekter

\footnotetext{
${ }^{31}$ VwGH vom 19. 3. 1921, Z. 1265/1921.

32 SZ IV/155.

${ }^{33}$ LENHOFF, Auflösung der Ehe 3.
} 
Kompetenzkonflikt wurde es für Dispensehegatten möglich, das auf Ungültigkeit der Dispensehe lautende Urteil durch eine Entscheidung des VfGH aufheben zu lassen. Üblicherweise erklärte die Mehrzahl der Gerichte die Dispensehe für ungültig, wurde der VfGH angerufen, so wurde das Urteil kassiert.

Im Anschluss daran ergänzte der $\mathrm{OGH}$ sein Gutachten aus 1921.34 Er bestätigte seine damalige Rechtsansicht und qualifizierte - ergänzend - die Erteilung der Nachsicht vom Ehehindernis des Ehebandes als "absolut nichtigen“, einer formellen, die Gerichte bindenden Rechtskraft nicht zugänglichen Verwaltungsakt. Bejahte aber der VfGH im Einzelfall einen positiven Kompetenzkonflikt und werde durch sein Erkenntnis eine für ungültig erklärte Dispensehe wieder hergestellt, so seien auch die Gerichte gebunden.

In der christlichsozialen Presse, insbesondere in der Reichspost, setzte eine massive Kampagne gegen den VfGH im Allgemeinen, Kelsen als ständigen Referenten im Besonderen ein. „Der Verfassungsgerichtshof gegen den Rechtsstaat eine bisher unübertroffene Groteske: Die Türken haben die Vielweiberei abgeschafft - der österreichische Verfassungsgerichtshof führt sie ein" lautet die Schlagzeile der Reichspost vom 9. November 1928. Kelsen wurde beschuldigt, der Bigamie Vorschub zu leisten und als "Haremswächter" karikiert. Unübersehbar waren bei dieser Pressekampagne gegen Kelsen die antisemitischen Untertöne. Auch wurde etwa die Wohnungstüre Kelsens mit Plakaten unflätigster sexueller Beschimpfungen beklebt. ${ }^{35}$

Der Konflikt wurde durch die „Umpolitisierung" des VfGH im Rahmen der VfGH Novelle 1929 "entschieden". 36

\footnotetext{
34 SZ 10/51.

${ }^{35}$ MÉTALL, Hans Kelsen 51.

${ }^{36}$ Vgl. NeschWARA, Entwicklung des Verfassungsrechts 140 .
}

Im Zuge der damit verbundenen Neubesetzung des VfGH änderte 1930 der VfGH seine Rechtsauffassung. Er verneinte „mangels Identität” der Sache einen Kompetenzkonflikt, womit ein auf Ungültigkeit der Dispensehe lautendes Urteil nicht mehr aufgehoben werden konnte.

Verschärft wurde der Ehewirrwarr überdies durch das burgenländische Eherecht. Mit der Angliederung des ehemals zu Ungarn gehörigen Burgenlandes ${ }^{37}$ wurde die Frage der Weitergeltung des ungarischen Eherechts akut. In Ungarn war seit 1894 die obligatorische Zivilehe verwirklicht, die Auflösung der Ehe („richterliche Lösung") stand allen Konfessionen unterschiedslos offen. Mit VO vom 29. Mai $1922^{38}$ sollten die „in Österreich geltenden Bestimmungen" des Eherechts und des Eheverfahrens mit 1. Jänner 1924 übernommen werden, sofern nicht vorher der burgenländische Landtag durch Beschluss die Beibehaltung der ungarischen Bestimmungen verlange. Dieser Beharrungsbeschluss für das ungarische Eherecht erfolgte am 12. Oktober 1922 mit den Stimmen der Sozialdemokraten, der Großdeutschen und des Bauernbundes und wurde verspätet vom Bundesminister für Justiz am 19. Dezember veröffentlicht. ${ }^{39}$ Diese burgenländische Sonderstellung führte zu bemerkenswerten Konstellationen des "interlokalen Privatrechts", denn u.a. kam das ungarische Recht im Sinne einer Reduktion von Umgehungsmöglichkeiten nur für jene Ehen zur Anwendung, bei denen der Ehemann (Bräutigam) die burgenländische Landesbürgerschaft vor dem 19. August 1921 oder nach diesem Zeitpunkt durch Geburt erworben hatte. ${ }^{40}$

\footnotetext{
${ }^{37}$ Staatsvertrag von St. Germain, StGBl. 1920/303, modifiziert durch die „Venediger Protokolle” vom 13. 10. 1921, BGBl. 1922/138.

38 BGBl. 315.

${ }^{39}$ Vgl. NeschWARA, Rezeption als Reform 39; IBY, Eherecht im Burgenland.

${ }^{40}$ BGBl. 1922/316.
} 
Versuchen, diesem Eherechtschaos mittels einer Reformgesetzgebung beizukommen, war kein Erfolg beschieden. Veränderungsanstrengungen gingen bis 1930 überwiegend von der Sozialdemokratie aus, ${ }^{41}$ sie waren jedoch alle im Hinblick auf die traditionellen kulturstaatlichen Frontstellungen zum Scheitern verurteilt. Adelheid Popp erkannte bald, dass eine grundsätzliche familienrechtliche Neuordnung im Sinne einer Gleichstellung von Mann und Frau in Verknüpfung mit einer Ehereform jedenfalls scheitern musste und brachte am 21. Juli 1925 unter ausdrücklicher Berufung auf die in der Verfassung verankerte Gleichstellung der Geschlechter ${ }^{42}$ einen familienrechtlichen Gleichstellungsantrag ein, der „zum ersten mal eine gänzliche Abkehr von der Geschlechtsrollenverteilung und die völlige Gleichstellung von Mann und Frau im Familienrecht [...] forderte“. ${ }^{43}$ Auch der elegante Vorstoß, die Großdeutschen mit der Forderung nach Übernahme oder zumindest Angleichung an das deutsche BGB-Eherecht zu einem Abgehen von der Koalitionsräson zu bewegen, blieb im Ergebnis erfolglos. ${ }^{44}$

Allerdings wurde eines deutlich. Die Reformanliegen gewannen zunehmend an parlamentarischem Einfluss. So wurde etwa unter Bundeskanzler Schober, der den Großdeutschen nahestand, einer Entschließung des Nationalrates folgend, Anfang 1930 ein Gesetzesentwurf ausgearbeitet, der, sieht man von Bestimmungen

\footnotetext{
${ }^{41} \mathrm{Vgl}$. den Überblick bei KREMSMAIR, Eherechtsreformversuche 515 .

42 FLOßMANN, Beschränkte Grundrechtssubjektivität 319.

${ }^{43}$ LEHNER, Familie - Recht - Politik 125; Abdruck des Antrags ebd. 575 (Anhang Nr. 2 b).

${ }^{44} 1929$ errangen die Ehereformer einen ersten Sieg, denn mit knapper Mehrheit (80:76) wurde ein Minderheitsantrag Severs angenommen. Die EntschlieBung forderte die Bundesregierung auf, dem Nationalrat ehebaldigst einen Entwurf über die Angleichung des österreichischen Eherechts an das deutsche vorzulegen; Sten. Prot. des NR, 3. GP, 77. vom 22. 1. 1929, 2220.
}

über Dispensehen $a b$, in den Grundzügen dem Entwurf von Staatssekretär Roller aus 1919 folgte. ${ }^{45}$ Der Christlichsozialen Partei wurde bewusst, dass bei den seit 1930 zunehmend prekären Mehrheitsverhältnissen im Nationalrat die aus ihrer Sicht - Gefahr für einen Erfolg eines entkonfessionalisierten Eherechts zunahm und sah im konkordatären Weg eine Möglichkeit, kultuspolitische Eckdaten zu verankern. ${ }^{46}$

\section{3. Konkordatseherecht}

Die Bemühungen um ein Konkordat sind in der Forschung bestens aufgearbeitet. Die Konkordatsverhandlungen, die bis in das Jahr 1929 zurückgehen, wurden von Dollfuß 1933 zügig zum Abschluss gebracht. Am 5. Juni 1933 wurde das Konkordat unterzeichnet, die Ratifikation erfolgte in der Nacht zum 1. Mai 1934, am 1. Mai 1934 trat das Konkordat mit der autoritären Verfassung in Kraft. ${ }^{47}$ Österreich erlebte während der Verhandlungen zwischen 1931 und 1934 mehrere Regierungswechsel, dementsprechend war auch die österreichische Haltung beträchtlichen Modifikationen unterworfen. Im Hinblick auf die notwendige parlamentarische Genehmigung wurde aber auf einen Text abgestellt, der die Option für eine zukünftige fakulta-

${ }^{45}$ Referierung bei KREMSMAIR, Eherechtsreformversuche 532.

${ }^{46}$ Am 13. 2. 1931 wurde mit 80 Stimmen der Sozialdemokraten und Großdeutschen gegen 79 Stimmen der Christlichsozialen, des Landbundes und des Heimatblockes ein Antrag auf Angleichung des österreichischen an das reichsdeutsche Eherecht angenommen - dies hätte die Übernahme der obligatorischen Zivilehe bedeutet; Sten Prot. des NR., VI. GP., vom 13.2.1931, 417. Auch im christlichsozialen Spektrum fanden sich Stimmen für eine Ehereform. Hervorzuheben sind etwa die Stellungnahmen des Kaplans Johann Kosnetter mit seinen „Gedanken zur Eherechtsreform in Österreich“, in denen er aus pastoralen Gründen für das „minus malum" einer Zivilehe eintrat; vgl. PRIMETSHOFER, KREMSMAIR, Gesetzliche Entwicklung 454f.; HANISCH, Bis dass der Tod euch scheidet 29.

${ }^{47}$ BGBl. II Nr. 1934/2. 
tive Zivilehe mit Ehetrennung für Katholiken, die sich nicht dem Regime des kanonischen Eherechts unterwerfen wollten, nicht verunmöglichte. ${ }^{48}$ Konsequent wird in Art. VII Konkordat die fakultative Zivilehe nicht erwähnt, aber auch nicht ausgeschlossen: So erkennt die Republik Österreich den nach kanonischem Recht abgeschlossenen Ehen die bürgerlichen Rechtswirkungen zu (§1). Die Zuständigkeit der kirchlichen Gerichte und Behörden zum Verfahren bezüglich der Ungültigkeit der Ehe und der Dispens von einer geschlossenen, aber nicht vollzogenen Ehe wird vom Staat anerkannt (§3), für den Eintritt der bürgerlichen Rechtswirkungen ist eine Vollstreckbarkeitserklärung durch den OGH notwendig. Im Zusatzprotokoll anerkannte Österreich auch die Zuständigkeit der kirchlichen Behörden zum Verfahren bezüglich des Privilegium Paulinum, der Hl. Stuhl konzedierte, dass das Verfahren bezüglich der Trennung von Tisch und Bett den staatlichen Gerichten zusteht (Zusatzprotokoll zu Art. VII Abs. 1 und 2). ${ }^{49}$

Die Zivilehe blieb als absolute Notzivilehe auch nach dem Konkordat bestehen. Katholische Nupturienten konnten ihrem zuständigen Pfarrer gegenüber einen kirchlichen Eheabschluss ablehnen, nach der formellen Abweisung durch das Trauungsorgan war die Möglichkeit einer Ziviltrauung eröffnet, allerdings, dem konfessionell geprägten Eherecht entsprechend, war auch diese Ehe nicht trennbar. Nur im Burgen-

\footnotetext{
${ }^{48}$ Hervorzuheben ist die Position von Max Hussarek, der vehement für die Zivilehe aus Gewissensgründen eintrat; zur symbolischen Bedeutung, am 1.5. die neue Verfassung in Kraft setzen zu lassen und das Konkordat zu ratifizieren vgl. HANISCH, Langer Schatten des Staates 310.

${ }^{49}$ Aus der reichhaltigen Literatur sei verwiesen auf: KREMSMAIR, Weg zum österreichischen Konkordat; DERS., Geschichte des österreichischen Konkordates 77 (mit Abdruck des kurialen Entwurfs vom 10. 8. 1931); WEINZIERL-FISCHER, Österreichische Konkordate 181ff., DIES., Konkordat von 1933119.
}

land mit der Vorgabe der obligatorischen Zivilehe bedingte das Konkordatseherecht ein System der fakultativen Zivilehe mit - so die Judikatur des $\mathrm{OGH}^{50}$ - Trennungsmöglichkeit dem Bande nach. Allerdings erließ die Bundesregierung 1935 ein Gesetz, das den staatlichen Gerichten untersagte, die katholischen burgenländischen Ehen dem Bande nach aufzulösen. ${ }^{51}$

Ein struktureller Vergleich mit dem neoabsolutistischen Konkordat liegt nahe ${ }^{52}$, doch müssen die Unterschiede mitberücksichtigt werden. Das Konkordat 1855 mit seinen kultuspolitischen Rahmenbedingungen revidierte die staatliche Ehehoheit für Katholikenehen, 1933 ging es "nur" um die Festigung der katholischen Ehe durch Aufwertung ihres Eheschließungsrechts.

Das Konkordatseherecht ${ }^{53}$ trug nichts zur Lösung der Eheprobleme bei, verschärfte vielmehr im Ergebnis die „Eherechtsunordnung” mit teilweise komplexen Fallgestaltungen. ${ }^{54}$ Die konkrete Anwendung des Konkordatseherechts blieb aber Episode, denn mit dem "Gesetz zur Vereinheitlichung des Rechts der Eheschließung und Ehescheidung im Land Österreich und im übrigen Reichsgebiet vom 6. Juli 1938“55 wurde eine neue Eherechtsgrundlage in Kraft gesetzt, die auch Ausgangspunkt für die Eherechtsentwicklung in der 2. Republik wurde.

\section{Das deutsche Ehegesetz}

Die Entstehungsgeschichte des Ehegesetzes ist gut dokumentiert: Grundsätzlich sollte das BGB - „volksfremd, gekünstelt (und) einer natürlichen Lebensanschauung zuwiderlaufend" -

\footnotetext{
${ }^{50}$ OGH vom 19. 5. 1935, 2 Ob 236/35 (JBl 1935, 498).

51 BGBl. 1935/134.

${ }^{52} \mathrm{HANISCH}$, Politischer Katholizismus, 77.

${ }^{53} \mathrm{Vgl}$. das Konkordats-Durchführungsgesetz vom 4. 5. 1934, BGBl. II, Nr. 1934/8.

${ }^{54}$ KÖSTLER, Österreichisches Konkordats-Eherecht; PRIMETSHOFER, Ehe und Konkordat, 27.

${ }^{55}$ DRGBl. I S 807; GBIÖ: 1938/244.
} 
durch ein Volksgesetzbuch ersetzt werden, eine Reform, die aber ein Torso blieb. Da jedoch der Bedarf zumindest eines zeitgemäßen Scheidungsrechts in der Bevölkerung weit verbreitet war, wurde eine derartige segmentäre Reform in Angriff genommen. Pointiert formuliert ging es um die Positionierung des Zerrüttungs- zu Lasten des Verschuldensprinzips, eingebettet in die NS Ehe- und Familienideologie. Radikal der Ausspruch, den der Münchener Rechtsanwalt Mößmer als Leiter des Familienausschusses der Akademie für Deutsches Recht in seiner Denkschrift 1935 erhob. Danach sollte eine Ehe ohne Verschulden geschieden werden können, wenn sie „für die ,Volksgemeinschaft' keinen Wert hatte", insbesondere, wenn aus ihr kein Nachwuchs zu erwarten war. Die Zerrüttung wurde objektiv formuliert, die subjektive Befindlichkeit der Ehepartner hatte zurückzutreten. So sollte eine Ehe auf Antrag des Staatsanwaltes zwangsweise aufgelöst werden, wenn "lebenswichtige Interessen der Gemeinschaft dauernd gröblich verletzt (waren)". Diesem - so Reichsjuristenführer Frank - „bisher bedeutsamsten und weltanschaulich geschlossensten Vorschlag" trat Justizminister Gürtner 1937 mit einem moderateren Entwurf entgegen. ${ }^{56}$

Eine unvorhergesehene Wende erfuhr die Reformdiskussion durch den Anschluss Österreichs. Hitler räumte der Ehebereinigung in Österreich hohe Priorität ein und erteilte am 22. Mai 1938 die Weisung, das Konkordat für Maßnahmen in Österreich als "nichtexistent“ zu behandeln. ${ }^{57}$ Damit wurde aber nicht nur eine Reform des Scheidungsrechtes notwendig, son-

\footnotetext{
${ }^{56}$ Vgl. BlasiUs, Ehescheidung in Deutschland194-209; GRUCHMANN, Ehegesetz 63-83; MERTENS, Rechtsetzung im Nationalsozialismus 46; ausgewählte Quellen bei SCHUBERT, Familien- und Erbrecht.

57 SCHOLDER, Österreichisches Konkordat 231f; zur rechtstheoretischen Begründung einer nachträglichen Rechtsgrundlage durch den Ausschuss für Religionsrecht der Akademie für Deutsches Recht vgl. RINNERTHALER, Ende des Konkordats 186.
}

dern auch des Eheschließungsrechtes, das Ergebnis: Das Ehegesetz vom 6. Juli 1938, wodurch das „altösterreichische Eherecht" quittiert wurde.

Ausgehend vom Entdeckungszusammenhang der österreichischen Eherechtsgeschichte bedeutete das "deutsche Ehegesetz" einen zentralen Einschnitt, einen Modernisierungs- und Säkularisierungsschub. Mit diesem Gesetz wurde die obligatorische Zivilehe eingeführt, Ehen konnten nur noch durch einen Standesbeamten "im Namen des Reiches" abgeschlossen werden. Das Eherecht war areligiös mit einem einer Kombination von Verschuldens- und Zerrüttungstatbeständen verpflichteten Scheidungsrecht. Das deutsche Ehegesetz beseitigte das österreichische Ehechaos, das einheitliche Scheidungsrecht - Scheidung im Sinne der Beseitigung des Ehebandes - machte die überkommene Terminologie von Trennung und Scheidung obsolet.

Dies war jedoch nur die eine Seite der Medaille, die andere, die düstere Seite, war die Übernahme völkisch-rassischer und eugenischer Bestimmungen im Kontext der Ehe- und Familienrechtsideologie des Nationalsozialismus. Intensive Berücksichtigung fand die NS-Rassenideologie im Ehehindernisrecht. Die Ehe, verstanden als Produktionsstätte erbgesunden arischen Nachwuchses führte zu spezifischen Eheverboten, Nichtigkeits- und Aufhebungsgründen, die der einschlägigen Rassenideologie und Erbgesundheitslehre entsprachen, hervorzuheben ist insbesondere die Berücksichtigung des Blutschutzgesetzes und seines rassenhygienischen Zwillings, des Ehegesundheitsgesetzes.

Im Ehescheidungsrecht ist - folgt man strikt dem Wortlaut - der direkte Konnex zur nationalsozialistischen Rassenideologie weniger augenfällig, hier waren aber generalklauselartige, unbestimmte Rechtsbegriffe wie „Wesen der Ehe”, die mittels „unbegrenzter Auslegung” (B. 
Rüthers) Interpretationen im Sinne der Volksgemeinschaftsideologie ermöglichten..$^{58}$

Nicht übersehen werden sollte, dass der österreichische Ehereformdiskurs der 20er Jahre für die Umsetzung eugenischer Regelungen anschlussfähig war.

So wurden zwar Anfang der 20er Jahre die letzen Residuen des politischen Ehekonsenses, einer Sozialkontrolle zur Verringerung der Anteile der subsistenzlosen Bevölkerung abgeschafft, ${ }^{59}$ zugleich aber versucht, eine eugenische und rassenhygienische Sozialkontrolle salonfähig zu machen. Empfahl beispielsweise der Tiroler Landtag im Interesse des allgemeinen Wohls die zwingende Überprüfung des Gesundheitszustandes von Ehewerbern mit Vorlage an die Behörde, so forderte weitaus unmissverständlicher der Landessanitätsrat im "roten” Wien Maßnahmen wie Zwangssterilisierungen und Zwangsasylierungen im Hinblick auf die Güte

\footnotetext{
${ }^{58}$ Vgl. den Scheidungsgrund der Fortpflanzungsverweigerung (§ 48EheG), der schon dem ALR wie BGB bekannt war, nun aber „,aus der bevölkerungspolitischen Zweckbestimmung der Ehe" motiviert wurde und nationalsozialistische erbgesundheitliche Reminiszenzen noch in der 2. Republik entfaltete; Fatale Anwendungsmöglichkeiten für die NS-Eheauffassung bot die Generalklausel des § 55 EheG. Die dadurch ermöglichte Scheidung nach dreijähriger Aufhebung der häuslichen Gemeinschaft räumte dem an der Zerrüttung schuldlosen Teil ein Widerspruchsrecht ein, das aber unbeachtlich war, wenn „die Aufrechterhaltung der Ehe bei richtiger Würdigung des Wesens der Ehe und des gesamten Verhaltens beider Ehegatten sittlich nicht gerechtfertigt war" - eine gravierende Einbruchsstelle für die NSIdeologie; Berüchtigt in seiner Wirkung auch der Aufhebungstatbestand des Eigenschaftsirrtums (§ 37 EheG); vgl. Blasius, Ehescheidung in Deutschland 210; RÜTHERS, Unbegrenzte Auslegung 400; HOFMEISTER, Privatrechtsgesetzgebung für Österreich 130; SCHAUER, Gesunde Nachkommenschaft 75.

${ }^{59}$ Beseitigung des politischen Ehekonsenses in Tirol mittels Landtagsbeschluss vom 28.1.1921, LGB1 1921/58 sowie in Vorarlberg durch Beschluss des Landtages vom 23. 3. 1923 (LGBl 1923/24), vgl. RINNERTHALER, Politischer Ehekonsens 297.
}

des Volksnachwuches. Wirkungsmächtig wurde das Konzept der "qualitativen Bevölkerungspolitik" des Wiener Stadtrates für das Wohlfahrtsund Gesundheitswesen Julius Tandler, um - so Tandler - den „biologischen Verfall des Volkes”, die drohende "Sintflut der Minderwertigen” aufzuhalten. ${ }^{60}$

Die "Metamorphosen“ des nationalsozialistischen Eherechts nach 1938 können im vorliegenden Zusammenhang übergangen werden, denn sie zeitigten für die Gesamtschau einer österreichischen Eheentwicklung keine relevante Wirkmächtigkeit, sondern entfalteten Bedeutung für das NS-Eherecht insbesondere im Kontext des Krieges. ${ }^{61}$

\section{Republik - Der Weg zur großen Familienrechtsreform}

Das R-ÜG vom 1. Mai 1945 sah die Aufhebung der nach dem 13. März 1938 erlassenen Gesetze und Verordnungen vor, "die mit dem Bestand des freien und unabhängigen Staates Österreich oder mit den Grundsätzen einer echten Demokratie unvereinbar sind, die dem Rechtsempfinden des österreichischen Volkes widersprechen oder typisches Gedankengut des Nationalsozia-

\footnotetext{
${ }^{60}$ Vgl. LeHNER, Verpönte Eingriffe 60; WOLF, Eugenische Vernunft 232.

${ }^{61}$ Z.B. die sog. "Stahlhelmehe“, im Volksmund „Leichentrauung" genannt. Aufgrund eines geheimen Führererlasses vom 6.7.1941 wurde der Reichsinnenminister ermächtigt, die nachträgliche Eheschließung mit gefallenen oder im Felde verstorbenen Wehrmachtsangehörigen anzuordnen, wenn die ernstliche Absicht zur Eheschließung bestanden hatte. Dazu korrespondierend wurde in der 5. DVEhG 1943 das Recht des toten Soldaten, sich von seiner "unwürdigen" Witwe scheiden zu lassen, verankert. Das Verfahren leitete der Staatsanwalt ein, der - so das Schwarze Korps der SS - „zum Anwalt der Sippe (wird), deren Recht und Ehre durch den Tod eines Gliedes nicht beeinträchtigt werden kann", vgl. EssNER, CONTE, Fernehe 201.
} 
lismus enthalten". ${ }^{62}$ Durch Kundmachung der Provisorischen Staatsregierung sollte festgestellt werden, welche Rechtsnormen als aufgehoben zu gelten haben ( $\$ 1$ Abs. 2), allerdings unterblieb eine derartige Kundmachung bezüglich des Ehegesetzes.

Ohne direkten Bezug auf das R-ÜG blieben aufgrund des Gesetzes vom 26. Juni 1945 über „Maßnahmen auf dem Gebiet des Eherechtes, des Personenstandsrechtes und des Erbgesundheitsrechtes" unter Eliminierung rassischbiologistischer Festlegungen das Ehegesetz inklusive Durchführungsverordnungen (mit Ausnahme der dritten) und das Personenstandsgesetz in Kraft. ${ }^{63}$

Die "Entnazifizierung" des Eherechts war aber nur teilweise gelungen, so waren die Streichungen in Deutschland umfangreicher, z.B. die Beseitigung des Scheidungsgrundes „Verweigerung der Fortpflanzung". Dieser erst 1999 aufgehobene Scheidungstatbestand "Verweigerung der Fortpflanzung" 64 sah als ein relevantes Tatbestandsmerkmal vor, dass der Ehegatte sich ohne triftigen Grund beharrlich weigert, Nachkommenschaft zu erzeugen oder zu empfangen. In der NS-Kommentierungsliteratur wurde der Begriff Nachkommenschaft mit „erbgesunden Kindern" identifiziert. In einigen einflussreichen zivilistischen Lehrbüchern und Kommentierungen bis in die 90er Jahre wurde die eugenische Grundvorstellung übernommen, allerdings wenigstens - „erbgesund” durch "gesund” ersetzt. 65

Diese und andere Spuren waren aber nicht Thema des eherechlichen Refomdiskurses der 2. Republik, denn hier wurden nochmals - Paradigma des zweiten konfessionellen Zeitalters die kultuspolitischen Bruchlinien mit einer geradezu stupenden Vehemenz aufgefahren.

\footnotetext{
62 StGBl. 1945/1.

${ }^{63}$ StGBl. 1945/31.

${ }^{64}$ Eherechts- ̈̈nderungsgesetz 1999, BGB1. I 125.

${ }^{65}$ Vgl. SCHAUER, Gesunde Nachkommenschaft 105.
}

Bis 1949 ist die Ehereformdiskussion vernachlässigbar. Wohl war die fakultative religiöse Eheschließung, insbesondere die Strafandrohung des §67 PStG für Seelsorger, die eine kirchliche Trauung vor der staatlichen vornahmen, Thema, doch war die Debatte wenig öffentlich wirksam und auch das Justizministerium engagierte sich kaum im Hinblick auf eine Familien- und Eherechtsreform. 66

1949 übernahm der Sozialist Otto Tschadek, praktizierender Katholik, das Justizressort und machte die Familienreform zu einem Schwerpunkt seiner Amtszeit. Tschadek scheiterte aber grandios, denn es verlagerte sich die Ehereformdiskussion in eine grundsätzliche kultuspolitische Debatte, in der es um eine Neupositionierung von Staat und Kirche ging.

1950 übermittelte Erzbischof Giovanni Dellepiane, seit 1949 Internuntius in Wien, eine diplomatische Note, mit der die Anerkennung des Konkordates eingemahnt wurde, eine Urgenz, die $\mathrm{zu}$ massiven Ablehnungen des „DollfußKonkordates" seitens der SPÖ führte. Den Startschuss machte Vizekanzler Schärf in der Monatszeitschrift "Zukunft". Er sah bereits in der Möglichkeit einer Wahlzivilehe eine verfehlte Gleichrangigkeit von Staat und Kirche. Nach Art. 15 StGG sei jede anerkannte Kirche den allgemeinen Staatsgesetzen unterworfen. Stelle man diese Subordinierung in Frage, führe dies zwingend zu einem neuen Verfassungskampf. Um die Weitergeltung des Konkordats und damit auch von Art. VII zu verhindern, bekennt sich Schärf zur Annexionstheorie, denn „die Propagierung der Okkupationstheorie führt dazu, das Konkordat für gültig zu erklären, ohne dass uns die Theorie sonst etwas nützt ${ }^{\prime \prime}{ }^{67}$ Der nun ausbrechende Konflikt, der zeitweise kulturkämpferische Ausmaße annahm, be-

\footnotetext{
${ }^{66} \mathrm{Vgl}$. POTZ, SCHINKELE, Kirchliche Trauung 404ff.

${ }^{67}$ Vgl. KLECATSKY, WeILER, Österreichisches Staatskirchenrecht 230; RAUCHENSTEINER, Die Zwei 316; PotZ, SCHINKELE, Kirchliche Trauung 411.
} 
stimmte die 50er Jahre und unterstreicht das Paradigma vom zweiten konfessionellen Zeitalter. Einen „kulturkämpferischen Höhepunkt“ markiert bereits das Jahr 1951. Eherechtsentwürfe, stark mitbestimmt von Professoren im Umkreis der 1949 gegründeten „Österreichischen Gesellschaft für Kirchenrecht" waren nicht mehrheitsfähig. Eine teilweise überzogene Rhetorik sowie juristische Einseitigkeiten von beiden Seiten ließen die von Tschadek am 19. November 1951 einberufene große Familienrechts Enquete "mit Getöse“ scheitern. ${ }^{68}$ So war die Verbindung der Forderung nach einer Wahlzivilehe und Anerkennung der Zuständigkeit der kirchlichen Gerichte und Behörden zur Durchführung von Verfahren bezüglich der Ungültigkeit der Ehe und der Dispens von einer geschlossenen, aber nicht vollzogenen Ehe bzw. die grundsätzliche Junktimierung mit der prinzipiellen Gültigkeit des Konkordats weder zwingend noch hilfreich. Auch der Verweis auf die Verfassungswidrigkeit der obligatorischen Zivilehe im Hinblick auf die Glaubens- und Gewissensfreiheit überzeugt nicht, derselbe Befund gilt für die Reduktion des § 67 PStG auf ausschließlich nationalsozialistisches Gedankengut. Demgegenüber litt aber auch der Einwand der Verfassungswidrigkeit der staatlichen Anerkennung kirchlich geschlossener Ehen an juristischer Stringenz.

Diese kultuspolitische Problemlage führte bereits 1952 zum Mariazeller Manifest mit seiner Absage an den politischen Katholizismus, ${ }^{69}$ doch die praktische Umsetzung dauerte an und forderte ein grundsätzliches Umdenken. Das bloße Einmahnen von staatlichen Verpflichtungen durch die Kirche, legitimiert durch eine von ihr verkündete Wahrheit, ging zunehmend an der Realität vorbei, auch die Vorstellung einer Partei als Partner im gesellschaftlichen Dialog und

\footnotetext{
${ }^{68}$ PotZ, SCHINKELE, Kirchliche Trauung 411.

${ }^{69} \mathrm{Vgl}$. SCHWARZ, Befreite Kirchen 289; LiEBMANN, Mariazeller Manifest 183.
}

nicht als bloßer Empfänger von kirchlichen Direktiven musste erst gelernt werden.

Ohne die Entwicklungen zu vertiefen: eine Versachlichung der Diskussionen bewirkte die Aufhebung von $\S 67$ PStG wegen Widerspruch zu Art. 15 StGG und Art. 63 Abs. 2 Vertrag von St. Germain durch den Verfassungsgerichtshof $1955^{70}$ sowie die Verlautbarung der Notenwechsel zwischen der österreichischen Bundesregierung und dem Päpstlichen Staatssekretariat am 6. März 1958 in der Wiener Zeitung. Daraus war zu entnehmen, dass die Fortgeltung des Konkordats in den vorher nicht abgeänderten oder aufgehobenen Bestimmungen von der österreichischen Bundesregierung anerkannt war. ${ }^{71}$ Auch die konkordatäre Neuregelung vermögens- und schulrechtlicher Fragen 1960 und $1962^{72}$ dokumentierte ein großes Maß an Kompromissbereitschaft seitens der SPÖ. Damit hatte sich die kultuspolitische Grundsatzdiskussion überlebt und der Ehereformdiskurs konnte ungeachtet unterschiedlicher ideologischer Positionierungen - ohne diesen kultuspolitischen Überbau mit seiner „Kampfrhetorik“ geführt werden.

Deutlich sichtbar wurden aber die unterschiedlichen Positionierungen am 1. Österreichischen Juristentag 1961. Hier erstattete Franz Bydlinski ein umfangreiches Gutachten zum Thema „Der Gleichheitsgrundsatz im österreichischen Pri-

\footnotetext{
70 VfGH Slg 2944/1955

${ }^{71}$ Vgl. KALB, Potz, SchinKele, Religionsrecht 454.

${ }^{72}$ Vertrag vom 23. 6. 1960, BGBl. 195, zur Regelung von vermögensrechtlichen Beziehungen (Vermögensvertrag); Vertrag vom 9. 7. 1962, BGBl. 273, zur Regelung von mit dem Schulwesen zusammenhängenden Fragen (Schulvertrag); anzuführen ist auch das kooperative konkordatäre Vorgehen bei den Diözesanerrichtungen (Diözesanerrichtungsverträge: Vertrag Eisenstadt, 23.6.1969, BGBl. 196; Vertrag Innsbruck, 7. 7. 1964, BGBl. 227; Vertrag Feldkirch, 7. 10. 1968, BGBl. 417); kennzeichnend für die religionsrechtliche Entspannung auch das Protestantengesetz 1961 (BGBl. 1961/12) und das Orthodoxengesetz 1967 (BGBl. 1967/229).
} 
vatrecht". Er versuchte engagiert den Gleichheitsgrundsatz im Familienrecht umzusetzen Stichworte: männliches Leitungsrecht, Namensrecht, Ehegüterrecht -, scheiterte aber wegen des damals vorherrschenden formalen Gleichheitsverständnisses. ${ }^{73}$

Sein Antipode war Franz Gschnitzer, tief verwurzelt in einem patriarchalisch-konservativen Familien- und Eheverständnis, der auch wesentlich für die Schlusserklärung der Tagung verantwortlich war: „Überlegungen de lege ferenda haben davon auszugehen, dass bereits unser ABGB sich grundsätzlich zur Gleichberechtigung bekannt und dort, wo es davon abgewichen ist, das aus sachlichen Erwägungen getan hat" ${ }^{\text {" }}{ }^{74}$

1962 führte der österreichische Anwaltstag eine umfangreiche inhaltliche Diskussion über einzelne Gebiete des Familien- und Eherechts, Bestandsaufnahme und Forderungen, die mit Ausgangspunkt für die legistischen Bemühungen der Jahre 1960-1970 war. Die Familienreform setzte aber erst mit der SPÖAlleinregierung 1970 unter Justizminister Broda ein, der auf Teilreformen abstellte. Erleichtert wurde der Reformkurs auch durch eine geänderte Haltung der ÖVP im Familienrecht, was sich z.B. im Salzburger Programm im Bekenntnis zur partnerschaftlichen Ehe niederschlug. ${ }^{75}$

Zentral für den Reformkurs war aber die sich verändernde Zivilgesellschaft. BürgerInnenbewegungen, StudentInnenbewegung und insbesondere die Neue Frauenbewegung weichten "die Dominanz des katholisch-konservativen sowie autoritär-sozialistischen Paradigmas" (E. Hanisch) auf, leiteten einen Bewusstseinswandel

\footnotetext{
${ }^{73}$ BYDLINSKI, Gutachten 79.

${ }^{74}$ GSCHNITZER, Referat 6; vgl. GASPERLMAIR, Die Diktatur geht 56; FLOßMANN, Frauenrechtsgeschichte 244.

${ }^{75}$ Vgl. LeHNER, Familie - Recht - Politik 192.
}

ein und führten zu einer „Normalisierung der säkularen Option“. ${ }^{76}$

Mit dem Reformgesetz 1975 war der Mann nicht mehr "Haupt der Familie”, nun galt, dass die „Rechte und Pflichten der Ehegatten im Verhältnis zueinander [...] gleich sind", dass die Ehegatten,$[\ldots]$ ihre eheliche Lebensgemeinschaft [...] einvernehmlich gestalten". ${ }^{77}$

1978 wurde mit der Aufhebung der $\S \S 1238$ 1241 ABGB die dem bürgerlichen Familienmodell verpflichtete Privilegierung des Mannes im Ehegüterrecht beseitigt und die einvernehmliche Scheidung vor dem Außerstreitrichter, bei der eine von beiden zugestandene Ehezerrüttung nicht weiter zu erforschen ist ( $\$ 55 a$ EheG), und die Beseitigung der Widerspruchslösung des an der Ehezerrüttung nicht oder minderschuldigen Ehegatten nach sechsjähriger Auflösung der ehelichen Gemeinschaft eingeführt. ${ }^{78}$ Damit war der Anfang gemacht, "ein patriarchales System, das lange Zeit einzig vorstellbar zu sein schien, zugunsten des Gedankens der Gleichrangigkeit der Geschlechter [...] aufzugeben“ (U. Floßmann). ${ }^{79}$

\footnotetext{
${ }^{76}$ JOAS, Wellen der Säkularisierung 726. Eine grundsätzliche ideologische Auseinandersetzung erfolgte in der Diskussion um die Straffreistellung eines Schwangerschaftsabbruches bis zum dritten Schwangerschaftsmonat (Fristenlösung; BGBl. 1974/60), doch wurde diese Debatte (mit durchaus entgleisender Rhetorik) weniger auf dem Hintergrund kultuspolitischer grundsätzlicher Rahmenbedingungen geführt, vgl. FloßmanN, Gusenleitner, Auswirkungen der Reformära 127; FLOßMANN, Frauenrechtsgeschichte 240.

${ }^{77}$ BG vom 1. 7. 1975, BGBl. 412, über die Neuordnung der persönlichen Rechtswirkungen der Ehe.

${ }^{78}$ BG vom 15. 6. 1978, BGBl. 280, mit Änderungen des Ehegattenerbrechts, des Ehegüterrechts und des Ehescheidungsrechts.

${ }^{79}$ FLOßMANN, Frauenrechtsgeschichte 251; Ausführliche und grundlegende Darstellung der Reform bei LEHNER, Familie - Recht - Politik 337 (Neuordnung der persönlichen Rechtswirkungen der Ehe), 455 (Reform des Ehegüter- und Ehegattenerbrechts), 511 (Reform des Ehescheidungsrechts).
} 


\section{Literatur:}

Klaus Berchtold (Hg.), Österreichische Parteiprogramme 1868-1966 (München 1967).

Olaf BLASCHKE, Das 19. Jahrhundert: Ein Zweites Konfessionelles Zeitalter?, in: Geschichte und Gesellschaft 26 (2000) 38-75.

DERS., Konfessionen im Konflikt. Deutschland zwischen 1800 und 1970: ein zweites konfessionelles Zeitalter (Göttingen 2002).

Dirk BLAsIUS, Ehescheidung in Deutschland 17451945. Scheidung und Scheidungsrecht in historischer Perspektive (Göttingen 1987).

John W. BOYER, Freud, Marriage and late Viennese Liberalism, in: The Journal of Modern History 50 (1978) 72-102.

Franz BYDLINSKI, Gutachten für den Ersten Österreichischen Juristentag, in: Vorstand des österreichischen Juristentages (Hg.), Verhandlungen des Ersten Österreichischen Juristentages I (Wien 1961).

Hermann CONRAD, Die Grundlegung der modernen Zivilehe durch die Französische Revolution. Ein Beitrag zur neueren Geschichte des Familienrechts, in: ZRG GA 67 (1950) 336-372.

DERS., Staatliche Theorie und kirchliche Dogmatik im Ringen um die Ehegesetzgebung Josephs II., in: Leo SCHEFFCZYK, Werner DETTLOFF, Richard HeInZManN (Hgg.), Festschrift Michael Schmaus, Bd. 2 (München 1967) 1172-1190.

Felix ERMACORA, Die Entstehung der Bundesverfassung 1929. Die Sammlung der Entwürfe zur Staats- bzw. Bundesverfassung (Wien 1990).

Cornelia EsSNER, Edouard CONTE, „Fernehe“, „Leichentrauung" und Totenscheidung. Metamorphosen des Eherechts um Dritten Reich, in: Vierteljahrshefte für Zeitgeschichte 44 (1996) 201-227.

Ursula FloßmanN, Karin GusenLeITNER, Die Auswirkungen der Reformära der 70er Jahre auf den Rechtswidrigkeitsbegriff des $\S 48$ (1) 2. Fall EheG 1938, in: Ursula FLOßMANN (Hg.), Nationalsozialistische Spuren im Recht. Ausgewählte Stolpersteine für ein selbstbestimmtes Frauenleben (Linz 1999) 127-174.

DIES., Die beschränkte Grundrechtssubjektivität der Frau. Ein Beitrag zum österreichischen Gleichheitsdiskurs, in: Ute GERHARD (Hg.), Frauen in der Geschichte des Rechts. Von der frühen Neuzeit bis zur Gegenwart (München 1997) 293-324.

DIES., Frauenrechtsgeschichte (Linz 2006).

DIES., Österreichische Privatrechtsgeschichte (Wien $\left.{ }^{6} 2008\right)$.

Elisabeth FRYSAK, Legale Kämpfe. Die petitionsrechtlichen Forderungen der österreichischen bürgerli- chen Frauenbewegung zur Änderung des Eheund Familienrechts um die Jahrhundertwende, in: L'homme. Zeitschrift für feministische Geschichtswissenschaft 14 (2003) 65-82.

Inken FUHRMANN, Die Diskussion über die Einführung der fakultativen Zivilehe in Deutschland und Österreich seit Mitte des 19. Jahrhunderts (Frankfurt am Main u.a. 1998).

Michaela GASPERLMAIR, Die Diktatur geht, die Demokratie kommt: Das Frauenbild bleibt gleich, in: Ursula FloßMANN (Hg.), Nationalsozialistische Spuren im Recht. Ausgewählte Stolpersteine für ein selbstbestimmtes Frauenleben (Linz 1999) 3-74.

Lothar GRUCHMANN, Das Ehegesetz vom 6. Juli 1938. Entstehung und Beurteilung, in: ZNR 11 (1989) 63-83.

Franz GsCHNITZER, Referat, in: Vorstand des österreichischen Juristentages (Hg.), Verhandlungen des Ersten Österreichischen Juristentages II (Wien 1961) 6-32.

Ernst HANISCH, Bis dass der Tod euch scheidet. Katholische Kirche und Ehegesetzgebung in Österreich, in: Erika WEINZIERL, Karl R. STADLER (Hgg.), Justiz und Zeitgeschichte. Geschichte der Familiengesetzgebung in Österreich (Wien 1977) 17-36.

DERS., Der lange Schatten des Staates. Österreichische Gesellschaftsgeschichte im 20. Jahrhundert (Wien 2005, ND der Ausgabe 1994).

DERS., Der Politische Katholizismus als ideologischer Träger des "Austrofaschismus", in: Emmerich TALós, Wolfgang Neugebauer (Hg.), Austrofaschismus. Politik - Ökonomie - Kultur (Wien ${ }^{52005)}$ 68-86.

Ulrike HARMAT, Ehe auf Widerruf? Der Konflikt um das Eherecht in Österreich 1918-1938 (Frankfurt am Main 1999).

Waltraud HEINDL, Frau und bürgerliches Recht. Bemerkungen zu den Reformvorschlägen österreichischer Frauenvereine vor dem ersten Weltkrieg, in: Isabella ACKERL, Walter HuMMELBERGER, Hans MOMMSEN (Hgg.), FS Neck (Wien 1981) 133-149.

Herbert HOFMEISTER, Privatrechtsgesetzgebung für Österreich unter der Herrschaft des Nationalsozialismus, in: Ulrike DAVY u.a. (Hgg.), Nationalsozialismus und Recht. Rechtssetzung und Rechtswissenschaft in Österreich unter der Herrschaft des Nationalsozialismus (Wien 1990) 124-148.

Paul IBY, Eherecht im Burgenland (1921-1938). Rechtsgeschichtliche Studie über ziviles und kirchliches Eherecht im Burgenland unter Einbeziehung der Geschichte des Eherechtes in Ungarn, (Eisenstadt 1990). 
Herbert KALB. Das kirchliche Ehenichtigkeitsverfahren in Österreich aus staatlicher Perspektive, in: Richard PuZA, Andreas WeIß (Hgg.), Festgabe Ernst Rößler (Frankfurt am Main u.a. 1997) 731743.

DERS., „Dispensehen“ und die Innsbrucker Juristenfakultät: Das Fakultätsgutachten vom 27. November 1919, in: Hans PAARHAMMER, Alfred RINNERTHALER (Hgg.), Österreich und der Heilige Stuhl im 19. und 20. Jahrhundert (Frankfurt am Main u.a. 2001) 273-292.

DERS., Richard POTZ, Brigitte SCHINKELE, Religionsrecht (Wien 2003).

Hans R. KlecAtSKY, Hans WeILER, Österreichisches Staatskirchenrecht (Wien 1958).

Rudolf KÖSTLER, Das österreichische KonkordatsEherecht (Wien 1937).

Josef KREMSMAIR, Der Weg zum österreichischen Konkordat von 1933/34 (Wien 1980).

DERS., Geschichte des österreichischen Konkordates 1933/34. Von den Anfängen bis zur Unterzeichnung, in: Hans PAARHAMMER, Franz POTOTSCHNIG, Alfred RinNERTHALER (Hgg.), 60 Jahre österreichisches Konkordat (München 1994), 77-118.

DERS., Die Eherechtsreformversuche in Österreich während der ersten Republik, in: Konrad BREITSCHING, Wilhelm REES (Hgg.), FS Mühlsteiger (Berlin 2001) 515-537.

Michael KÜHnLEIN, Matthias LutZ-BaChMAnN (Hg.), Neue Perspektiven auf das Werk von Charles Taylor (Berlin 2011).

Karin LEHNER, Verpönte Eingriffe. Sozialdemokratische Reformbestrebungen zu den Abtreibungsbestimmungen in der Zwischenkriegszeit (Wien 1989).

Oskar LeHNER, Familie - Recht - Politik. Die Entwicklung des österreichischen Familienrechts im 19. und 20. Jahrhundert (Wien-New York 1987).

Arthur LENHOFF, Auflösung der Ehe und Wiederverehelichung (Wien 1926).

DERS., in: Heinrich KLANG, Kommentar zum Allgemeinen bürgerlichen Gesetzbuch, Bd. 1, Halbbd. 1 (Wien 1933).

Peter LEISCHING, Die römisch-katholische Kirche in Cisleithanien, in: Adam WANDRUSZKA, Peter URBANITSCH (Hgg.), Die Habsburgermonarchie 18481918, Bd. 4: Die Konfessionen (Wien 1985) 1-247.

Maximilian LIEBMANN, Das Mariazeller Manifest, in: Heimo KAINDL, Alois RUHRI (Hgg.), Thron und Altar. 1000 Jahre Staat und Kirche (Graz 1996) 183191.

Bernd MERTENS, Rechtsetzung im Nationalsozialismus (Tübingen 2009).
Rudolf Aladár MÉTALL, Hans Kelsen. Leben und Werk (Wien 1969).

Christian NeschWARA, Rezeption als Reform. Das ungarische Eherecht im österreichischen Burgenland 1921, in: ZNR 11 (1989) 39-62.

DERS., Zur Entwicklung des Verfassungsrechts nach 1918, in: Herbert SCHAMBECK (Hg.), Parlamentarismus und öffentliches Recht in Österreich. Entwicklung und Gegenwartsprobleme, Teilbd. 1, (Berlin 1993) 83-219.

DERS., Hans Kelsen und das Problem der Dispensehen, in: Thomas OlechOWSKI, Werner OGRIS (Hgg.), Hans Kelsen. Leben - Werk - Wirksamkeit (Wien 2009) 246-263.

DERS., Kelsen als Verfassungsrichter. Seine Rolle in der Dispensehen-Kontroverse, in: Stanley PAULSON, Michael STOLleIs (Hgg.), Hans Kelsen Staatsrechtslehrer und Rechtstheoretiker (Tübingen 2005) 353-384.

Christa PEILIKAN, Aspekte der Geschichte des Ehrerechts in Österreich (Wien 1980).

Richard POTZ, Brigitte SchINKELE, Die kirchliche Trauung im staatlichen Recht Österreichs. Ein Beitrag zur Geschichte des Verhältnisses von Staat und Katholischer Kirche in den langen Fünfzigerjahren, in: Hans PAARHAMMER, Alfred RinNERTHALER (Hgg.), Österreich und der Heilige Stuhl im 19. und 20 Jh. (Frankfurt am Main u.a. 2001) 401-442.

Franz РототsсHNIG, Staatlich-kirchliche Ehegesetzgebung im 19. Jahrhundert (Instructio Austriaca) (Wien 1974).

Bruno PRIMETSHOFER, Ehe und Konkordat (Rom 1960).

Bruno PrIMETSHOFER, Josef KREMSMAIR, Die gesetzliche Entwicklung der Beziehungen von Kirche und Staat, in: Herbert ScHAMBECK (Hg.), Parlamentarismus und öffentliches Recht in Österreich. Entwicklung und Gegenwartsprobleme, Teilbd. 1 (Berlin 1993) 397-471.

Manfried RAUCHENSTEINER, Die Zwei. Die Große Koalition in Österreich 1945-1966 (Wien 1987).

Alfred RINNERTHALER, Der politische Ehekonsens im Herzogtum Salzburg, in: Salzburg. Geschichte \& Politik 2 (1992) 259-304.

DERS., Das Ende des Konkordats und das Schicksal wichtiger Konkordatsmaterien in der NS-Zeit, in: Hans PAARHAMmer, Franz POTOTSCHNiG, Alfred RINNERTHALER (Hgg.), 60 Jahre österreichisches Konkordat (München 1994) 179-229.

DERS., Johann Kirchsteiger und seine „Salzburger Ehen", in: Ulrike AICHORN, Alfred RINNERTHALER (Hgg.), FS Peter Putzer, Bd. 2 (Egling an der Paar 2004) 823-867. 
Helmut RUMPLER, Eine Chance für Mitteleuropa. Bürgerliche Emanzipation und Staatsverfall in der Habsburgermonarchie (Wien 2005, ND. d. Ausgabe 1994).

Bernd RÜTHERS, Die unbegrenzte Auslegung. Zum Wandel der Privatrechtsordnung im Nationalsozialismus (Tübingen ${ }^{62005) .}$

Martin SCHAUER, Das Recht der Ehescheidung im 19. und 20. Jahrhundert. Vom Verschuldensprinzip zum Zerrüttungsprinzip (iur. Diss., Univ. Linz 1998).

DERS., Die "gesunde Nachkommenschaft" von der NS-Zeit bis heute. Ein Beitrag zum österreichischen Scheidungsrecht, in: Ursula FLOßMANN (Hg.), Nationalsozialistische Spuren im Recht. Ausgewählte Stolpersteine für ein selbstbestimmtes Frauenleben (Linz 1999) 75-125.

Klaus SCHOLDER, Österreichisches Konkordat und nationalsozialistische Kirchenpolitik 1938/39, in: ZevKR 20 (1975) 230-243.
Werner SCHUBERT, Das Familien- und Erbrecht unter dem Nationalsozialismus. Ausgewählte Quellen (Paderborn u.a. 1993).

Karl SchWARZ, Befreite Kirchen im freien Staat. Zur Religionspolitik der Republik Österreich in den 50er Jahren, in: ÖAKR 44 (1988) 289-314.

DERS., Rezension von HARMAT, Ehe auf Widerruf?, in: ZRG KA 88 (2002) 502-512.

Erika WEINZIERL-FISCHER, Die österreichischen Konkordate von 1855 und 1933 (Wien 1960).

Erika WEINZIERL, Das österreichische Konkordat von 1933. Von der Unterzeichnung bis zur Ratifikation, in: Hans PAARHAMMER, Franz POTOTSCHNIG, Alfred RinNERTHALER (Hgg.), 60 Jahre österreichisches Konkordat (München 1994) 119-134.

Maria A. Wolf, Eugenische Vernunft. Eingriffe in die reproduktive Kultur durch die Medizin 1900-2000 (Wien-Köln-Weimar 2008).

Benjamin ZiEMANN, Säkularisierung und Neuformierung des Religiösen. Gesellschaft und Religion in der zweiten Hälfte des 20. Jahrhunderts, in: Archiv für Sozialgeschichte 51 (2011) 3-36. 